\title{
FUZZY PROCESSING ON GPS DATA TO IMPROVE THE POSITION ACCURACY
}

\author{
Chung-Jie Lin, Yung-Yaw Chen, Fan-Ren Chang \\ Department of Electric Engineering . National Taiwan University, \\ Taipei, Taiwan, 10764 , R.O.C \\ TEL : 886-2-363-5251 EXT 241 \\ FAX: 886-2-363-8247 \\ E-mail : frchang@ac.ee.ntu.edu.tw
}

ABSTRACT

A new application of fuzzy set theory to the problem of GPS positioning accuracy improvement is presented. We employed fuzzy processing on the $\mathrm{C} / \mathrm{A}$ code stand alone receiver and the DGPS receiver. The membership functions for the processing are determined by position dilution of precision (PDOP), signal to noise ratio (SNR) and the reliable factor of fixed position. We can select the more accurate position fixes according to the values of reliable factors. The accuracy of positioning has been improved by selecting position fixes from the original ones.

\section{Introduction}

Since Zadeh's proposal of the fuzzy set theory in 1960's, fuzzy logic has been widely and successfully, applied to lots of researches. The application of fuzzy theory to reduce the GPS position error seems to be an interesting topic. The position accuracy of the GPS receiver is very important to the users, especially for those who use the C/A code stand alone receiver. Thus, the fuzzy processing on GPS data to improve the position accuracy is proposed.

In this paper, the relationship between PDOP, SNR of the code and position error is discussed. Then the concept of this processing and experiment results are shown in the followings. It will illustrate that fuzzy processing on GPS data can actually reduce the position error to a certain extent.

\section{Basic Concepts}

\subsection{PDOP and SNR}

The accuracy with which positions are determined using GPS mostly depends on two factors: the satellite configuration geometry, and the measurement accuracy. The usual term for GPS measurement accuracy is the user equivalent range error (UERE), which represents the combined effect of ephemeris uncertainties. propagation errors, clock and timing errors, and receiver noise.

For 3D positioning purpose, four satellites in view are needed. The effect of satellite configuration geometry is expressed by the dilution of precision (DOP) 
factor, which is the ratio of the positioning accuracy to the measurement accuracy, or

$$
\sigma=\mathrm{DOP} * \sigma_{0}
$$

where $\sigma_{0}$ is the measurement accuracy (standard deviation), and

$\sigma$ is the positioning accuracy

DOP will change with time, as the satellites travel alone their orbits, and from place to place, since the satellite configuration is position-dependent. The PDOP is defined as the DOP value for the 3-D position and we want the PDOP to be as small as possible. An example of coordination of satellites which gives a minimum PDOP is shown in Fig. 1.

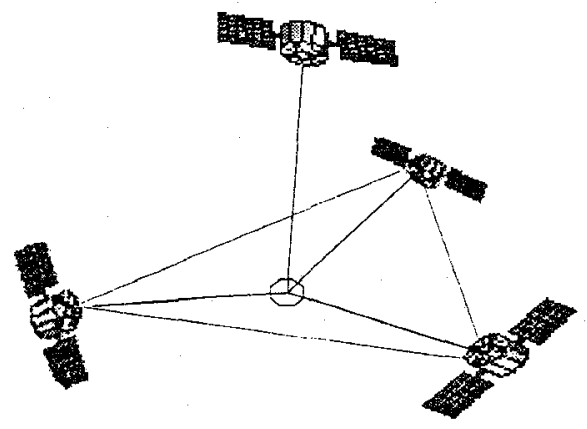

Fig. 1. Geometry configuration of satellites for a minimum PDOP

The user has a zenith satellite and the other three satellites with low elevation angle. This configuration gives small POP value. However, ionosphere and troposphere disturbance affect the signals from these low elevation angle satellites, much more than the signal from higher angle satellite. Furthermore, when the satellite elevation angle become lower, the SNARE become smaller. The small SNARE of the specific satellites' means that large pseudo range error exists. Even if, the relationship between the SNARE and pseudo range error is not clear, there is still tradeoffs among the POP and SNARE. The relationship between them possesses fuzziness, and that is the reason why we use fuzzy processing on GPs data to enhance the positioning accuracy.

\subsection{The Architecture of the Processing}

The block diagram of GPS fuzzy processing is shown in Fig. 2. Two MOTOROLA SIXGUN-620 GPS receivers and a personal computer are used in this experiment. 
The PDOP and the sum of SNR of three satellites excluding a zenith satellite, were used to evaluate the reliable factor, which represents the reliability of this position fix, i.e. latitude, longitude, and height.

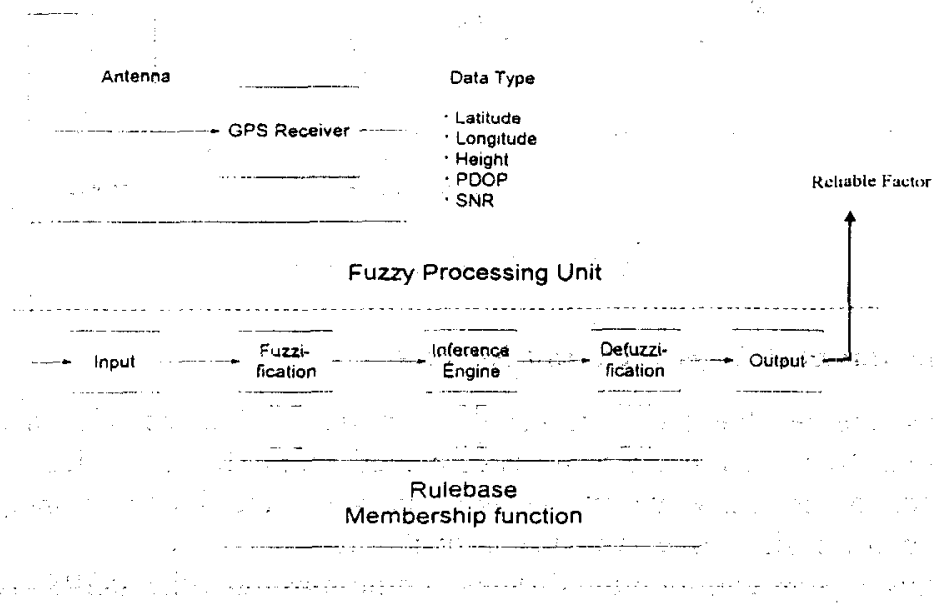

Fig. 2. The block diagram of GPS fuzzy processing

\section{Fuzzy Processing}

The PDOP and the sum of SNR are used as input fuzzy variables to the fuzzy processing unit, and the variables are respectively divided into three and four segments to partition the rule space. The reliable factor is the output fuzzy variable fuzzied with a singleton membership function. The approximate reasoning method is used for the inference process and the center of area method is employed for the defuzzification. There are twelve rules in the rule base as follows:

(1) IF PDOP is large and the sum of SNR is zero THEN Reliable factor is zero

(2) IF PDOP is large and the sum of SNR is small THEN Reliable factor is zero.

(3) IF PDOP is large and the sum of SNR is medium THEN Reliable factor is zero.

(4) IF PDOP is large and the sum of SNR is large THEN Reliable factor is small

(5) IF PDOP is medium and the sum of SNR is zero THEN Reliable factor is zero.

(6) IF PDOP is medium and the sum of SNR is small THEN Reliable factor is zero.

(7) IF PDOP is medium and the sum of SNR is medium THEN Reliable factor is small.

(8) IF PDOP is medium and the sum of SNR is large THEN Reliable factor is medium.

(9) IF PDOP is small and the sum of SNR is zero THEN Reliable factor is zero,

(10) IF PDOP is small and the sum of SNR is small THEN Reliable factor is small.

(11) IF PDOP is small and the sum of SNR is medium TIIEN Reliable factor medium.

(12) IF PDOP is small and the sum of SNR is large THEN Reliable factor is large. 
The membership functions are shown in Fig. 3.
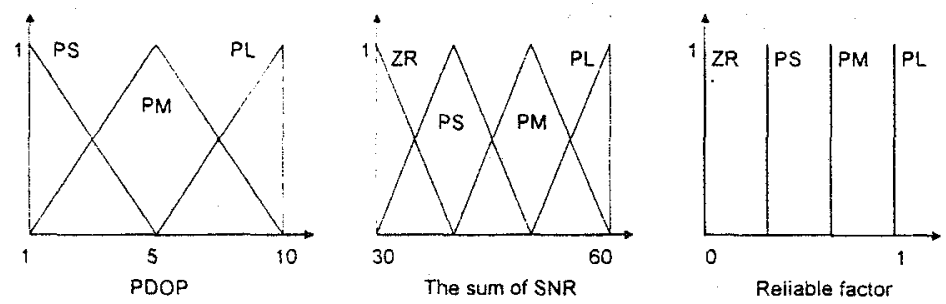

Fig. 3. Membership functions

\section{Experimental Approach and Results}

Thus, the reliable factor of the position fix is obtained after the fuzzy processing. Next the reliable factor is compared with a desired critical value. If it exceeds the critical value, this position fix is adopted. By this principle, we can select the position fixes from the original ones.

In this experiment, both the C/A code stand alone and the DGPS receivers are used to verify the position error improved by the fuzzy processing. As shown in Fig. 4 and Fig. 5, the original 1000 position fixes were collected on the building in the National Taiwan University ( N25'01'10.542", E121 $1^{\circ} 32^{\prime} 26.895^{\prime \prime}$, 13:22'-13:39', May 10, 1996).

The number of fuzzy-selected position fixes is 600 . To compare fuzzied position fix with minimum PDOP fix and maximum SNR fix, we select the position fixes with the minimal PDOP and the maximal sum of SNR, respectively. The results for the stand alone GPS receiver and DGPS receivers are shown as the following :

\begin{tabular}{|c||c|c|c||c|c|c|}
\hline \multicolumn{1}{||}{} & \multicolumn{3}{c|}{ Stand alone receiver } & \multicolumn{3}{c|}{ DGPS receivers } \\
\cline { 2 - 7 } & $\sigma($ Lat) & $\sigma($ Lon $)$ & $\sigma(2 \mathrm{D})$ & $\sigma$ (Lat) & $\sigma$ (Lon) & $\sigma(2 \mathrm{D})$ \\
\hline $\begin{array}{c}\text { Original } \\
\text { Data }\end{array}$ & $16.57 \mathrm{~m}$ & $27.33 \mathrm{~m}$ & $31.96 \mathrm{~m}$ & $0.6184 \mathrm{~m}$ & $0.8063 \mathrm{~m}$ & $1.0161 \mathrm{~m}$ \\
\hline $\begin{array}{c}\text { Futa: - } \\
\text { Selected }\end{array}$ & $8.59 \mathrm{~m}$ & $11.9 \mathrm{~m}$ & $14.67 \mathrm{~m}$ & $0.2794 \mathrm{~m}$ & $0.6523 \mathrm{~m}$ & $0.7096 \mathrm{~m}$ \\
\hline Max-STR & $8.8 \mathrm{~m}$ & $12.1 \mathrm{~m}$ & $15.01 \mathrm{~m}$ & $0.3120 \mathrm{~m}$ & $0.7062 \mathrm{~m}$ & $0.7718 \mathrm{~m}$ \\
\hline Min-PDOP & $19.8 \mathrm{~m}$ & $36.04 \mathrm{~m}$ & $\mathbf{4 1 . 1 2 \mathrm { m }}$ & $0.6550 \mathrm{~m}$ & $0.7291 \mathrm{~m}$ & $0.9801 \mathrm{~m}$ \\
\hline
\end{tabular}

Table 1. Summary of experiment results 
From Table 1., the results have shown that the positioning errors strongly depend on the sum of SNR. On the other hand, the PDOP value does not affect the error strongly. The fuzzy-selected data have the smallest $\sigma$ 's, although not too much different from the Max-SNR approach.

\section{Conclusions}

As shown in the results ,the GPS position accuracy was improved by the fuzzy processing. The future work is that introducing neural networks into the GPS receiver to improve positioning errors, e.g. Selective Availability (SA) effect, which is the main error source for the C/A code stand alone receiver.

\section{References}

[1] Leick, A. . GPS satellite surveying. Wiley, New York Chichester Brisbane Toronto Singapore. 1990

[2] B. Hofmann-Wellenhof, H. Lichtenegger, and J. Collins, Global Positioning System : theory and practice. New York: Springer-Verlag, 1992.

[3] B. Kosko. Neural Networks and Fuzzy Systems. Englewood Cliffs, NJ: Prentice-Hall, 1992.

[4] Kazunobu Koremura, Michihiro Asakura, and Chiaki Matsumoto, "Position accuracy improvement using fuzzy processing on GPS data," Proc. ION-GPS-94.

[5] D. Dubois. and H. Prade. "Fuzzy sets in approximate reasoning, part 1: Inference with possibility distributions," Fuzzy Sets Syst. vol. 40. 1991, pp. 143-202.

[6] H. J. Zimmermann, Fuzzy Sets Theory and its Applications, Boston: Kjuwer-Nijhoff Publishing. 1992
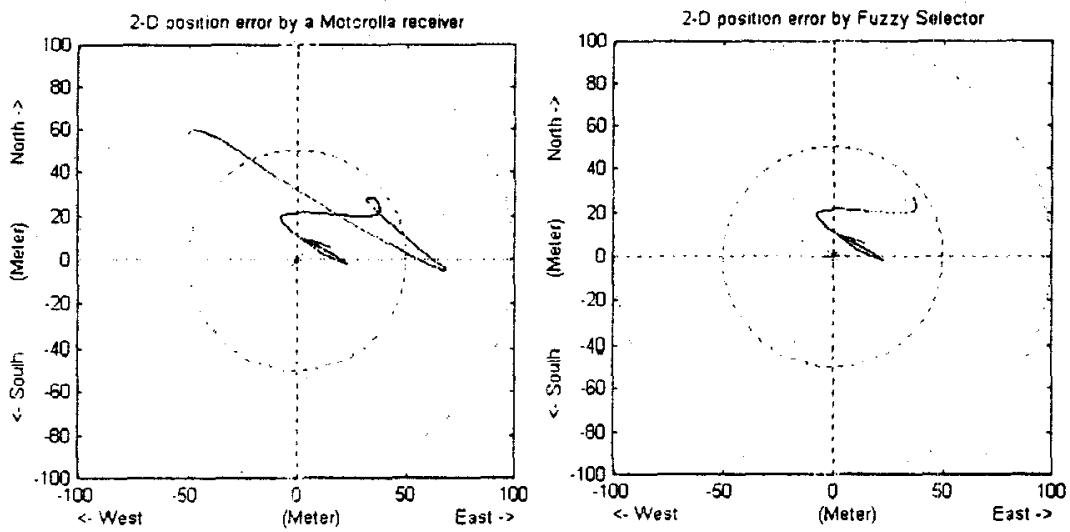

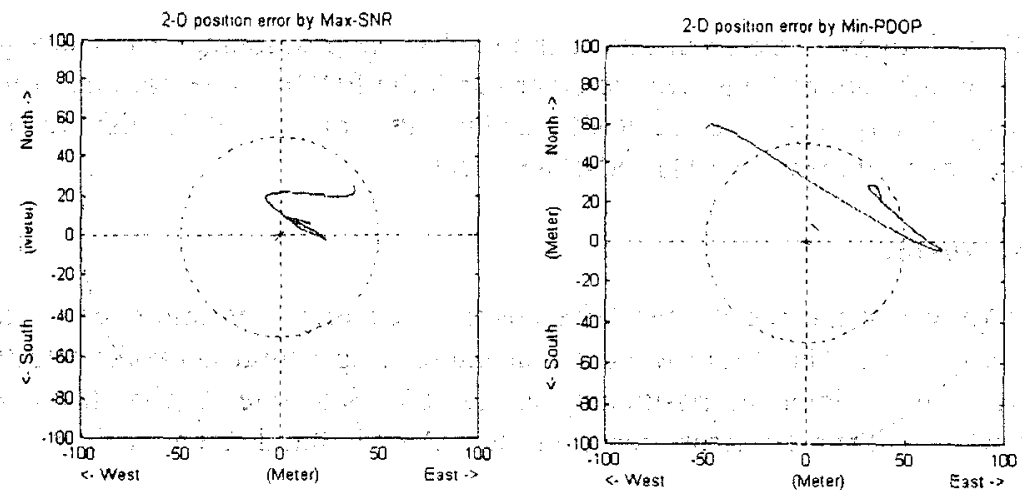

Fig. 4. The results for the stand alone receiver
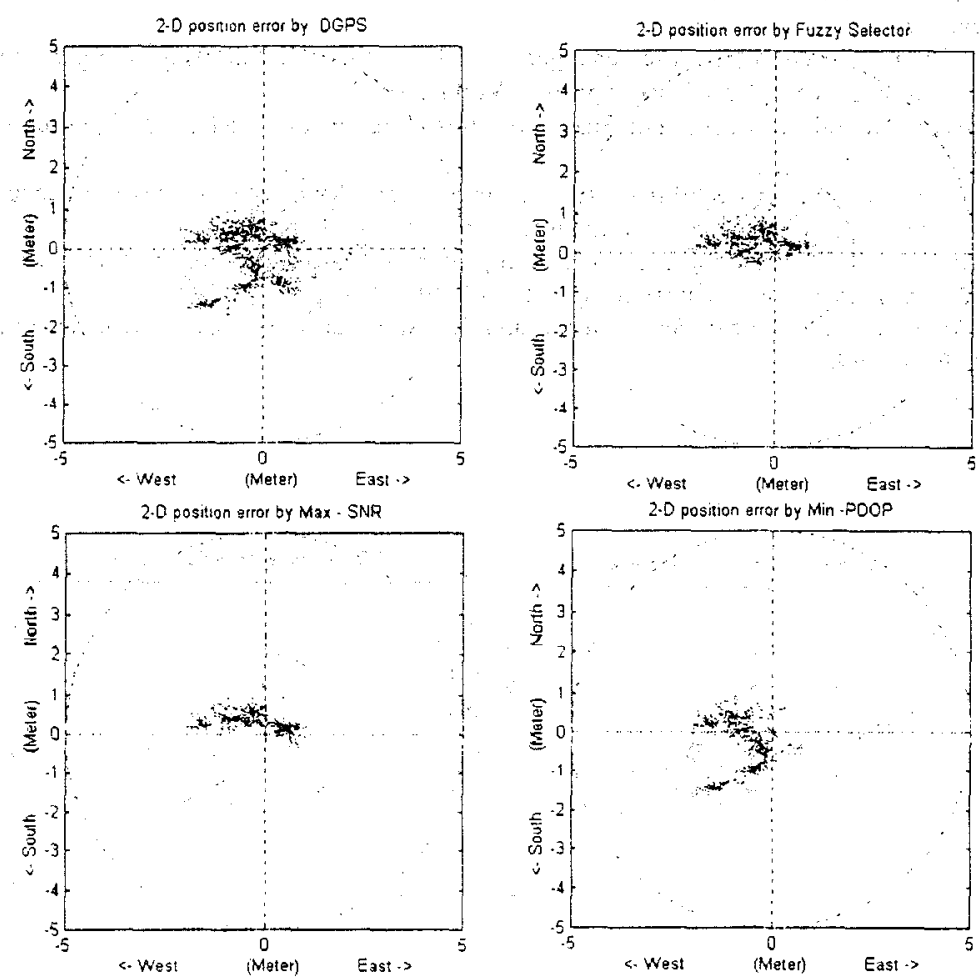

Fig. 5. The results for the DGPS receivers 